DESY 07-094

\title{
Dispersion representations for hard exclusive processes: beyond the Born approximation
}

\author{
M. Diehl ${ }^{1}$ and D. Yu. Ivanov ${ }^{2}$ \\ 1 Theory Group, Deutsches Elektronen-Synchroton DESY, 22603 Hamburg, Germany \\ 2 Sobolev Institute of Mathematics, 630090 Novosibirsk, Russia
}

\begin{abstract}
Several hard exclusive scattering processes admit a description in terms of generalized parton distributions and perturbative hard-scattering kernels. Both the physical amplitude and the hard-scattering kernels fulfill dispersion relations. We give a detailed investigation of their consistency at all orders in perturbation theory. The results shed light on the information about generalized parton distributions that can be extracted from the real and imaginary parts of exclusive amplitudes. They also provide a practical consistency check for models of these distributions in which Lorentz invariance is not exactly satisfied.
\end{abstract}

\section{Introduction}

Dispersion relations play an important role in the description of exclusive processes, relating the real and imaginary parts of the amplitude. They are for instance required to derive the operator product expansion for Compton scattering in Bjorken kinematics. In this context they have recently been used to establish a representation of the deeply virtual Compton amplitude which allows the inclusion of two-loop corrections in a practicable way [1. In a different context, dispersion relations have been employed in [2] to simplify the calculation of the hard-scattering kernels for exclusive quarkonium production at next-to-leading order.

For hard exclusive processes that can be calculated using collinear factorization, one may write down dispersion relations both for the physical process and for the parton-level subprocess. The question of consistency between both representations turns out to be nontrivial and has already been 
raised in the seminal work [3] giving the proof of factorization for meson production. Important progress has recently been reported in [4, where it was shown that this consistency is ensured by Lorentz invariance in the form of the polynomiality property for generalized parton distributions (GPDs). The studies in [4] were carried out using the Born-level approximation of the hard-scattering subprocess. In particular, they showed that to this accuracy not only the imaginary but also the real part of the process amplitude can be represented in terms of GPDs $F(x, \xi, t)$ along the line $x=\xi$ in the $x-\xi$ plane. This constitutes both a simplification and a limitation for extracting information on GPDs from hard exclusive amplitudes at leading-order accuracy. It is natural to ask how the situation changes when including radiative corrections to the hard-scattering kernel.

In the present work we therefore investigate dispersion representations for hard exclusive processes to all orders in perturbation theory, generalizing the leading-order results derived in [4]. In addition

we consider in detail the distributions for polarized quarks and for gluons, for which special issues arise. Our paper is organized as follows. In the next section we recall a number of results which will be needed in our subsequent work. Section 3 gives a detailed analysis of dispersion representations in the unpolarized quark sector. The specifics of other distributions are discussed in Sect. 4. As an application of our results, we investigate in Sect. 5 the model for GPDs proposed by McDermott, Freund and Strikman [5], where polynomiality is not satisfied. In Sect. 6] we summarize our findings and draw conclusions.

\section{Some reminders}

Let us begin by recalling some well-known properties of generalized parton distributions and of dispersion relations, which we will need in the subsequent sections.

\subsection{Lorentz invariance and crossing properties}

An essential property of generalized parton distributions is the polynomiality of their Mellin moments. This property directly follows from the Lorentz covariance of the operator matrix elements which are parameterized by GPDs [6]. With the conventional definitions (given e.g. in [7]) we have for quarks

$$
\begin{aligned}
\int_{-1}^{1} d x x^{n-1} H^{q}(x, \xi, t) & =\sum_{\substack{k=0 \\
\text { even }}}^{n-1}(2 \xi)^{k} A_{n, k}^{q}(t)+(2 \xi)^{n} C_{n}^{q}(t), \\
\int_{-1}^{1} d x x^{n-1} E^{q}(x, \xi, t)= & \sum_{\substack{k=0 \\
\text { even }}}^{n-1}(2 \xi)^{k} B_{n, k}^{q}(t)-(2 \xi)^{n} C_{n}^{q}(t), \\
\int_{-1}^{1} d x x^{n-1} \widetilde{H}^{q}(x, \xi, t)= & \sum_{\substack{k=0 \\
\text { even }}}^{n-1}(2 \xi)^{k} \widetilde{A}_{n, k}^{q}(t), \\
\int_{-1}^{1} d x x^{n-1} \widetilde{E}^{q}(x, \xi, t)= & \sum_{\substack{k=0 \\
\text { even }}}^{n-1}(2 \xi)^{k} \widetilde{B}_{n, k}^{q}(t)
\end{aligned}
$$

with $n \geq 1$, where $C_{n}^{q}$ is nonzero only for even $n$. For gluons we have 


$$
\begin{aligned}
\int_{0}^{1} d x x^{n-2} H^{g}(x, \xi, t) & =\sum_{\substack{k=0 \\
\text { even }}}^{n-2}(2 \xi)^{k} A_{n, k}^{g}(t)+(2 \xi)^{n} C_{n}^{g}(t), \\
\int_{0}^{1} d x x^{n-2} E^{g}(x, \xi, t) & =\sum_{\substack{k=0 \\
\text { even }}}^{n-2}(2 \xi)^{k} B_{n, k}^{g}(t)-(2 \xi)^{n} C_{n}^{g}(t), \\
\int_{0}^{1} d x x^{n-2} \widetilde{H}^{g}(x, \xi, t) & =\sum_{\substack{k=0 \\
\text { even }}}^{n-1}(2 \xi)^{k} \widetilde{A}_{n, k}^{g}(t), \\
\int_{0}^{1} d x x^{n-2} \widetilde{E}^{g}(x, \xi, t) & =\sum_{\substack{k=0 \\
\text { even }}}^{n-1}(2 \xi)^{k} \widetilde{B}_{n, k}^{g}(t),
\end{aligned}
$$

where $n \geq 2$ is even for $H^{g}$ and $E^{g}$ and $n \geq 3$ is odd for $\widetilde{H}^{g}$ and $\widetilde{E}^{g}$. Since $H^{g}, E^{g}$ are even and $\widetilde{H}^{g}, \widetilde{E}^{g}$ are odd functions of $x$, we can restrict the integrals in (2) to the range $0<x<1$. The convention for the moment index $n$ is such that quark and gluon form factors with the same $n$ mix under evolution, i.e. $A_{n, k}^{q}$ with $A_{n, k}^{g}, B_{n, k}^{q}$ with $B_{n, k}^{g}$ etc. The different powers of $x$ in the integrals (11) and (2) reflect the different forward limits of the distributions, e.g. $H^{q}(x, 0,0)=q(x)$ and $H^{g}(x, 0,0)=x g(x)$ for $x>0$.

An important ingredient in the subsequent discussion will be the high-energy behavior of scattering amplitudes. According to the principles of Regge theory, this behavior is connected with the quantum numbers exchanged in the $t$-channel. Let us briefly recall how the relevant quantum numbers can be determined in the context of generalized parton distributions [8]. For negative or zero $t$ the form factors $A_{n, k}^{q}(t)$ etc. parameterize the matrix elements of quark or gluon operators between single-proton states. Their analytic continuation to positive $t$ gives the corresponding matrix elements between the vacuum and a proton-antiproton state. Decomposing those matrix elements into contributions with definite angular momentum, one can associate the form factors with the relevant quantum numbers in the $t$-channel. The relevant decomposition for the GPDs of the proton is given in Chapt. 4.2 of [7], and we list the resulting $J^{P C}$ quantum numbers of the $t$-channel exchange in Table 1. From this one can readily establish the exchange quantum numbers for the generalized parton distributions, which are given in Table 2. In particular we see that for positive charge conjugation there are distributions allowing for spin-zero exchange. This corresponds to energy independent contributions in scattering amplitudes, which play a prominent role in dispersion relations as we will see.

A way to ensure polynomiality of the moments (1) is the double distribution representation $[9,8$,

$$
H^{q}(x, \xi, t)=H_{f}^{q}(x, \xi, t)+\operatorname{sign}(\xi) D^{q}\left(\frac{x}{\xi}, t\right), \quad E^{q}(x, \xi, t)=E_{k}^{q}(x, \xi, t)-\operatorname{sign}(\xi) D^{q}\left(\frac{x}{\xi}, t\right)
$$

with

$$
\begin{aligned}
& H_{f}^{q}(x, \xi, t)=\int d \beta d \alpha \delta(x-\alpha \xi-\beta) f^{q}(\beta, \alpha, t), \\
& E_{k}^{q}(x, \xi, t)=\int d \beta d \alpha \delta(x-\alpha \xi-\beta) k^{q}(\beta, \alpha, t),
\end{aligned}
$$


Table 1: Quantum numbers of $t$-channel exchanges for the form factors in (1) and (2) as explained in the text. The entries with positive charge conjugation parity $C=+1$ refer to both quarks gluons, and those with $C=-1$ only to quarks.

\begin{tabular}{lrr}
\hline \hline \multicolumn{1}{c}{ form factor } & \multicolumn{1}{c}{$n$} \\
\hline$A_{n, k}+\frac{t}{4 m^{2}} B_{n, k}$ & even & $0^{++}, 2^{++}, \ldots,(n-k)^{++}$ \\
$C_{n}$ & even & $0^{++}$ \\
$A_{n, k}+B_{n, k}$ & even & $2^{++}, \ldots,(n-k)^{++}$ \\
$\widetilde{A}_{n, k}+\frac{t}{4 m^{2}} \widetilde{B}_{n, k}$ & odd & $0^{-+}, 2^{-+}, \ldots,(n-k-1)^{-+}$ \\
$\widetilde{A}_{n, k}$ & odd & $1^{++}, 3^{++}, \ldots,(n-k)^{++}$ \\
\hline$A_{n, k}+\frac{t}{4 m^{2}} B_{n, k}$ & odd & $1^{--}, 3^{--}, \ldots,(n-k)^{--}$ \\
$A_{n, k}+B_{n, k}$ & odd & $1^{--}, 3^{--}, \ldots,(n-k)^{--}$ \\
$\widetilde{A}_{n, k}+\frac{t}{4 m^{2}} \widetilde{B}_{n, k}$ & even & $1^{+-}, 3^{+-}, \ldots,(n-k-1)^{+-}$ \\
$\widetilde{A}_{n, k}$ & even & $2^{--}, \ldots,(n-k)^{--}$ \\
\hline \hline
\end{tabular}

Table 2: Quantum numbers of $t$-channel exchanges for combinations of generalized quark distributions of definite charge conjugation parity. The entries with $C=+1$ also hold for the corresponding gluon distributions.

\begin{tabular}{ll}
\hline \hline distribution & \multicolumn{1}{c}{$J^{P C}$} \\
\hline$H^{q}(x, \xi, t)-H^{q}(-x, \xi, t)$ & $0^{++}, 2^{++}, \ldots$ \\
$E^{q}(x, \xi, t)-E^{q}(-x, \xi, t)$ & $0^{++}, 2^{++}, \ldots$ \\
$\widetilde{H}^{q}(x, \xi, t)+\widetilde{H}^{q}(-x, \xi, t)$ & $1^{++}, 3^{++}, \ldots$ \\
$\widetilde{E}^{q}(x, \xi, t)+\widetilde{E}^{q}(-x, \xi, t)$ & $0^{-+}, 1^{++}, 2^{-+}, 3^{++}, \ldots$ \\
\hline$H^{q}(x, \xi, t)+H^{q}(-x, \xi, t)$ & $1^{--}, 3^{--}, \ldots$ \\
$E^{q}(x, \xi, t)+E^{q}(-x, \xi, t)$ & $1^{--}, 3^{--}, \ldots$ \\
$\widetilde{H}^{q}(x, \xi, t)-\widetilde{H}^{q}(-x, \xi, t)$ & $2^{--}, 4^{--}, \ldots$ \\
$\widetilde{E}^{q}(x, \xi, t)-\widetilde{E}^{q}(-x, \xi, t)$ & $1^{+-}, 2^{--}, 3^{+-}, 4^{--}, \ldots$ \\
\hline \hline
\end{tabular}


where $f^{q}$ and $k^{q}$ are commonly referred to as double distributions and $D^{q}$ as the $D$-term. The support region of $f^{q}(\beta, \alpha, t)$ and $k^{q}(\beta, \alpha, t)$ is the rhombus $|\alpha|+|\beta| \leq 1$, whereas $D^{q}(\alpha, t)$ has support for $|\alpha|<1$ and is odd in $\alpha$. More general representations have been discussed in the literature [8, 10, 11] but will not be needed in the following. For gluons one has

$$
H^{g}(x, \xi, t)=H_{f}^{g}(x, \xi, t)+|\xi| D^{g}\left(\frac{x}{\xi}, t\right), \quad E^{g}(x, \xi, t)=E_{k}^{g}(x, \xi, t)-|\xi| D^{g}\left(\frac{x}{\xi}, t\right)
$$

with

$$
\begin{aligned}
& H_{f}^{g}(x, \xi, t)=\int d \beta d \alpha \delta(x-\alpha \xi-\beta) \beta f^{g}(\beta, \alpha, t), \\
& E_{k}^{g}(x, \xi, t)=\int d \beta d \alpha \delta(x-\alpha \xi-\beta) \beta k^{g}(\beta, \alpha, t) .
\end{aligned}
$$

The support properties of $f^{g}, k^{g}$ and $D^{g}$ are as for their quark counterparts, and $D^{g}(\alpha, t)$ is even in $\alpha$. One readily finds that the Mellin moments of the $D$-term are related to the form factors $C_{n}(t)$ as

$$
\int_{-1}^{1} d \alpha \alpha^{n-1} D^{q}(\alpha, t)=2^{n} C_{n}^{q}(t), \quad \quad \int_{0}^{1} d \alpha \alpha^{n-2} D^{g}(\alpha, t)=2^{n} C_{n}^{g}(t) .
$$

The polarized quark distributions $\widetilde{H}^{q}$ and $\widetilde{E}^{q}$ have double distribution representations analogous to (3) and (4) but without a $D$-term, since the highest power appearing in their Mellin moments (11) is $\xi^{n-1}$ instead of $\xi^{n}$. We will discuss the case of $\widetilde{H}^{g}$ and $\widetilde{E}^{g}$ in Section 4.3 .

\subsection{Dispersion relations}

The exclusive processes we consider in this work are deeply virtual Compton scattering (DVCS) and light meson production,

$$
\gamma^{*}(q)+p(p) \rightarrow \gamma\left(q^{\prime}\right)+p\left(p^{\prime}\right), \quad \gamma^{*}(q)+p(p) \rightarrow M\left(q^{\prime}\right)+p\left(p^{\prime}\right),
$$

where four-momenta are indicated in parentheses. Our arguments can be extended to the production of heavy mesons like the $J / \Psi$, but we shall not dwell on this here. Since the processes in (8) involve particles with nonzero spin, the appropriate quantities for discussing dispersion relations are invariant amplitudes, which have simple analyticity and crossing properties. An explicit decomposition for Compton scattering can be found in [12, where these invariant amplitudes are called Compton form factors.

To describe the kinematics of (8) we use the Mandelstam variables $s=(p+q)^{2}, t=\left(p-p^{\prime}\right)^{2}$, $u=\left(p-q^{\prime}\right)^{2}$. Consider now an invariant amplitude $\mathcal{F}^{[\sigma]}(\nu, t)$ with definite signature $\sigma$ under $s \leftrightarrow u$ crossing, so that

$$
\mathcal{F}^{[\sigma]}(-\nu, t)=\sigma \mathcal{F}^{[\sigma]}(\nu, t)
$$

where $2 \nu=s-u$. We will work in kinematics where $t \leq 0$ and external photons are on shell or have spacelike virtuality, so that the imaginary part of the amplitude is due to the $s$-channel discontinuity for $\nu>0$ and to the $u$-channel discontinuity for $\nu<0$. The fixed- $t$ dispersion relation with no subtraction then reads

$$
\operatorname{Re} \mathcal{F}^{[\sigma]}(\nu, t)=\frac{1}{\pi} \int_{\nu_{t h}}^{\infty} d \nu^{\prime} \operatorname{Im} \mathcal{F}^{[\sigma]}\left(\nu^{\prime}, t\right)\left[\frac{1}{\nu^{\prime}-\nu}+\sigma \frac{1}{\nu^{\prime}+\nu}\right],
$$


where $\nu_{t h}$ is the value of $\nu$ at threshold. Here and in the following Cauchy's principal value prescription is understood for the singularities at $\nu^{\prime}= \pm \nu$ of the dispersion integral. For the dispersion relation (10) to be valid, the integral of $\mathcal{F}^{[\sigma]}\left(\nu^{\prime}, t\right)$ times the term in square brackets must vanish when taken over an infinite semicircle in the $\nu^{\prime}$ plane. This requires

$$
\mathcal{F}^{[+]}(\nu, t) \underset{|\nu| \rightarrow \infty}{\rightarrow} 0, \quad \quad \nu^{-1} \mathcal{F}^{[-]}(\nu, t) \underset{|\nu| \rightarrow \infty}{\rightarrow} 0 .
$$

A dispersion relation with one subtraction,

$$
\begin{aligned}
& \operatorname{Re} \mathcal{F}^{[\sigma]}(\nu, t)-\operatorname{Re} \mathcal{F}^{[\sigma]}\left(\nu_{0}, t\right) \\
& \quad=\frac{1}{\pi} \int_{\nu_{t h}}^{\infty} d \nu^{\prime} \operatorname{Im} \mathcal{F}^{[\sigma]}\left(\nu^{\prime}, t\right)\left[\frac{1}{\nu^{\prime}-\nu}+\sigma \frac{1}{\nu^{\prime}+\nu}-\frac{1}{\nu^{\prime}-\nu_{0}}-\sigma \frac{1}{\nu^{\prime}+\nu_{0}}\right],
\end{aligned}
$$

is valid if

$$
\nu^{-2} \mathcal{F}^{[+]}(\nu, t) \underset{|\nu| \rightarrow \infty}{\rightarrow} 0
$$

whereas for $\sigma=-1$ we have the same condition (11) as with no subtraction.

We will study dispersion relations for the processes (8) in the Bjorken limit of large $-q^{2}$ at fixed $q^{2} / \nu$ and $t$. It is then useful to trade $\nu$ for the scaling variable

$$
\xi=-\frac{\left(q+q^{\prime}\right)^{2}}{2\left(p+p^{\prime}\right) \cdot\left(q+q^{\prime}\right)}=-\frac{q^{2}}{s-u}=-\frac{q^{2}}{2 \nu},
$$

where we have neglected $q^{2}$ and $t$ compared with $q^{2}$ in the numerator. The factorization theorems established in [3, 13, state that in the Bjorken limit certain invariant amplitudes become dominant and can be written as the convolution of partonic hard-scattering kernels with generalized quark or gluon distributions (and the light-cone distribution amplitude of the produced meson) 1 To establish dispersion relations we will need information on the high-energy behavior of these amplitudes. Empirically the small- $x$ behavior of the usual quark and gluon distributions, obtained from fits mainly to inclusive deep inelastic scattering data, is well described by a power law. With currently used models for generalized parton distributions, based either on double distributions or on Gegenbauer moments, one finds a corresponding power-law behavior for the invariant amplitudes of DVCS [12, 14, 15, 1] and of meson production [16]. Whether this correspondence may be model-independent is not known, see the discussion in Sect. 3.2 of [15]. We will take it as a guideline in the following, bearing in mind that deviations between the power laws of parton densities and exclusive amplitudes (or deviations from a strict power behavior in the asymptotic limit) do not invalidate our dispersion relations as long as the invariant amplitudes do not grow faster than the critical power of energy specified in (11) and (13).

\section{Unpolarized quark distributions}

In this section we discuss in detail the contribution of unpolarized quark distributions to the leading invariant amplitudes for DVCS or meson production. Here and in the following we decompose all

\footnotetext{
${ }^{1} \mathrm{Up}$ to terms suppressed by inverse powers of $\sqrt{-q^{2}}$, the leading invariant amplitudes for DVCS correspond to transverse photon polarization and those for meson production to longitudinal photon and meson polarization in the collision c.m.
} 
amplitudes into terms of definite signature $\sigma$. According to the factorization theorem we can write

$$
\mathcal{F}^{q[\sigma]}\left(\xi, t, q^{2}\right)=\int_{-1}^{1} d x \frac{1}{\xi} C^{q[\sigma]}\left(\frac{x}{\xi}, q^{2}\right) F^{q}(x, \xi, t)
$$

with $F^{q}=H^{q}, E^{q}$. For simplicity we have omitted the dependence on the renormalization and factorization scales; in the following will also omit the arguments $q^{2}$ in the hard-scattering kerne $2^{2}$ and $t$ in the generalized parton distributions. The hard-scattering kernel satisfies the symmetry relation

$$
C^{q[\sigma]}\left(-\frac{x}{\xi}\right)=-\sigma C^{q[\sigma]}\left(\frac{x}{\xi}\right)
$$

so that the factorization formula can be written as

$$
\mathcal{F}^{q[\sigma]}(\xi)=\int_{0}^{1} d x \frac{1}{\xi} C^{q[\sigma]}\left(\frac{x}{\xi}\right) F^{q[\sigma]}(x, \xi)
$$

in terms of the combinations

$$
F^{q[\sigma]}(x, \xi)=F^{q}(x, \xi)-\sigma F^{q}(-x, \xi)
$$

for quark exchange of definite signature. We remark that $F^{q[+]}$ corresponds to positive and $F^{q[-]}$ to negative charge conjugation parity in the $t$-channel. With the relation

$$
F^{q[\sigma]}(x,-\xi)=F^{q[\sigma]}(x, \xi)
$$

from time reversal invariance one finds $\mathcal{F}^{q[\sigma]}(-\xi)=\sigma \mathcal{F}^{q[\sigma]}(\xi)$ as required. In the Bjorken limit the Mandelstam variables for the hard-scattering subprocess are given by

$$
\hat{s}=x s+\frac{1}{2}(1-x) q^{2}, \quad \hat{u}=x u+\frac{1}{2}(1-x) q^{2},
$$

so that one has

$$
\frac{x}{\xi}=-\frac{\hat{s}-\hat{u}}{q^{2}} .
$$

To leading order $(\mathrm{LO})$ in $\alpha_{s}$ the kernel reads

$$
C^{q[\sigma]}(\omega) \propto \frac{1}{1-\omega-i \epsilon}-\sigma \frac{1}{1+\omega-i \epsilon}, \quad \operatorname{Im} C^{q[\sigma]}(\omega) \propto \pi[\delta(\omega-1)-\sigma \delta(\omega+1)]
$$

for both DVCS and meson production, where we have omitted any global factors which are irrelevant for our discussion of fixed- $t$ dispersion relations here. At higher orders in $\alpha_{s}$ one finds branch cuts in the $\hat{s}$ and $\hat{u}$ channels for $\omega>1$ and $\omega<-1$, respectively. For the dispersion relations to be discussed shortly, we need to know the behavior of the kernels for $|\omega| \rightarrow \infty$. The NLO kernels for DVCS can be found in [17], and those for meson production in [18]. For negative signature, one finds $C^{q[-]}(\omega) \sim \omega^{-1}$ up to logarithms for both DVCS and meson production. For positive signature, the NLO corrections give $C^{q[+]}(\omega) \sim \omega^{-1}$ for DVCS, and $C^{q[+]}(\omega) \sim \omega^{0}$ for meson production, again up to logarithms. The power behavior as $\omega^{0}$ is due to two-gluon exchange in the $t$-channel. For DVCS such graphs only start at NNLO, so that at this level one will also have $C^{q[+]}(\omega) \sim \omega^{0}$. This change in energy behavior between NLO and NNLO is the same as in the hard-scattering kernels for inclusive deep inelastic scattering [19], obtained from $\gamma^{*} p \rightarrow \gamma^{*} p$ in forward kinematics via the optical theorem. In fact, the kernels for DVCS and for deep inelastic scattering are intimately related, see e.g. [1, 17].

\footnotetext{
${ }^{2}$ We refer to $C^{q[\sigma]}$ as hard-scattering kernel for ease of language, keeping in mind that for meson production it is more precisely the convolution of a hard-scattering kernel with the meson distribution amplitude.
} 


\subsection{Dispersion relations}

The invariant amplitude satisfies a fixed- $t$ dispersion relation. Using $1 / \xi=-2 \nu / q^{2}$ as energy variable and making one subtraction, one has

$$
\operatorname{Re} \mathcal{F}^{q[\sigma]}(\xi)-\operatorname{Re} \mathcal{F}^{q[\sigma]}\left(\xi_{0}\right)=\frac{1}{\pi} \int_{1}^{\infty} d \omega^{\prime} \operatorname{Im} \mathcal{F}^{q[\sigma]}\left(1 / \omega^{\prime}\right)\left[\frac{1}{\omega^{\prime}-1 / \xi}+\sigma \frac{1}{\omega^{\prime}+1 / \xi}-\left\{\xi \rightarrow \xi_{0}\right\}\right]
$$

where $\xi_{0}$ denotes the subtraction point and the Cauchy principal value prescription is understood at $\omega^{\prime}= \pm 1 / \xi$. As is appropriate in the Bjorken limit, we have neglected $t$ and the hadron masses when determining the lower limit of the $\omega^{\prime}$ integration.

According to the discussion at the end of the previous section, the validity of a dispersion relation with one subtraction requires that $\xi^{2} \mathcal{F}^{q[+]}(\xi)$ and $\xi \mathcal{F}^{q[-]}(\xi)$ vanish for $\xi \rightarrow 0$, whereas an unsubtracted dispersion relation would require $\mathcal{F}^{q[+]}(\xi) \rightarrow 0$ in the same limit. Given the phenomenological observed small- $x$ behavior of valence and sea quark distributions, we expect a small- $\xi$ behavior $\mathcal{H}^{q[\sigma]}(\xi) \sim \xi^{-\alpha}$ with $1<\alpha<2$ for $\sigma=+1$ and $0<\alpha<1$ for $\sigma=-1$. For $\sigma=+1$ we hence do require one subtraction in the dispersion relation. We have also taken one subtraction for $\sigma=-1$ although this would not be necessary. We shall see that our final results for negative signature would be the same with no subtraction. According to Table 2 the distributions $H^{q[\sigma]}$ and $E^{q[\sigma]}$ involve the same quantum numbers in the $t$-channel, and we therefore expect that the high-energy behavior of $\mathcal{H}^{q[\sigma]}$ and $\mathcal{E}^{q[\sigma]}$ is similar.

Inserting the factorization formula (17) into (23) and using that $C^{q[\sigma]}(\omega)$ has a vanishing imaginary part for $|\omega|<1$, one obtains

$$
\begin{aligned}
\operatorname{Re} & \mathcal{F}^{q[\sigma]}(\xi)-\operatorname{Re} \mathcal{F}^{q[\sigma]}\left(\xi_{0}\right) \\
= & \frac{1}{\pi} \int_{1}^{\infty} d \omega^{\prime} \int_{1 / \omega^{\prime}}^{1} d x \omega^{\prime} \operatorname{Im} C^{q[\sigma]}\left(x \omega^{\prime}\right) F^{q[\sigma]}\left(x, 1 / \omega^{\prime}\right)\left[\frac{1}{\omega^{\prime}-1 / \xi}+\sigma \frac{1}{\omega^{\prime}+1 / \xi}-\left\{\xi \rightarrow \xi_{0}\right\}\right] \\
= & \frac{1}{\pi} \int_{1}^{\infty} d \omega \int_{0}^{1} d x \frac{\omega}{x^{2}} \operatorname{Im} C^{q[\sigma]}(\omega) F^{q[\sigma]}\left(x, \frac{x}{\omega}\right)\left[\frac{1}{\omega / x-1 / \xi}+\sigma \frac{1}{\omega / x+1 / \xi}-\left\{\xi \rightarrow \xi_{0}\right\}\right],
\end{aligned}
$$

where from the second to the third line we have changed the order of integration, $\int_{1}^{\infty} d \omega^{\prime} \int_{1 / \omega^{\prime}}^{1} d x=$ $\int_{0}^{1} d x \int_{1 / x}^{\infty} d \omega^{\prime}$, substituted $\omega=x \omega^{\prime}$, and changed the order of integration again. Straightforward algebra finally gives

$$
\begin{aligned}
& \operatorname{Re} \mathcal{F}^{q[\sigma]}(\xi)=\operatorname{Re} \mathcal{F}^{q[\sigma]}\left(\xi_{0}\right) \\
& \quad+\frac{1}{\pi} \int_{1}^{\infty} d \omega \operatorname{Im} C^{q[\sigma]}(\omega) \int_{0}^{1} d x F^{q[\sigma]}\left(x, \frac{x}{\omega}\right)\left[\frac{1}{\omega \xi-x}-\sigma \frac{1}{\omega \xi+x}-\frac{1}{\omega \xi_{0}-x}+\sigma \frac{1}{\omega \xi_{0}+x}\right]
\end{aligned}
$$

Note that $\operatorname{Im} C^{q[\sigma]}(\omega)$ contains terms proportional to $\delta(\omega-1)$, as is already seen in the leadingorder expression (22). These terms are understood to be included in the integration over $\omega$ in (25). A remark is in order on the behavior of the integrand for $x \rightarrow 0$. Let us first consider the case $F^{q[\sigma]}=H^{q[\sigma]}$. It is natural to expect that $H^{q[\sigma]}(x, x / \omega)$ has a singular behavior for $x \rightarrow 0$ that is similar to the forward distribution $q(x)+\sigma \bar{q}(x)$. With the small- $x$ behavior of quark densities obtained in typical phenomenological analyses, one then has an integrable singularity of $H^{q[-]}(x, x / \omega)$, whereas the corresponding singularity of $H^{q[+]}(x, x / \omega)$ is stronger than $x^{-1}$ but weaker than $x^{-2}$. For $\sigma=+1$ the expression in square brackets in (25) is however proportional to $x$, so that the integrand is again sufficiently well behaved at $x=0$. A similar discussion can be given for $E^{q[\sigma]}(x, x / \omega)$, assuming that its small- $x$ behavior is similar to the one of $H^{q[\sigma]}(x, x / \omega)$. 
We now discuss the dispersion relation for the hard-scattering kernel itself. Notice that according to (16) the kernel $C^{q[\sigma]}$ has opposite symmetry behavior under crossing than the corresponding process amplitude $\mathcal{F}^{q[\sigma]}$, so that $C^{q[+]}$ satisfies a negative-signature dispersion relation and $C^{q[-]}$ a positivesignature one. With the large- $\omega$ behavior discussed after (22) we hence need no subtraction in either case and can write

$$
\operatorname{Re} C^{q[\sigma]}\left(\frac{x}{\xi}\right)=\frac{1}{\pi} \int_{1}^{\infty} d \omega \operatorname{Im} C^{q[\sigma]}(\omega)\left[\frac{1}{\omega-x / \xi}-\sigma \frac{1}{\omega+x / \xi}\right],
$$

where again the Cauchy principal value prescription is implied at $\omega= \pm x / \xi$. Insertion into the factorization formula (17) yields

$$
\operatorname{Re} \mathcal{F}^{q[\sigma]}(\xi)=\frac{1}{\pi} \int_{1}^{\infty} d \omega \operatorname{Im} C^{q[\sigma]}(\omega) \int_{0}^{1} d x F^{q[\sigma]}(x, \xi)\left[\frac{1}{\omega \xi-x}-\sigma \frac{1}{\omega \xi+x}\right] .
$$

This can in particular be used to evaluate the subtraction constant $\operatorname{Re} \mathcal{F}^{q[\sigma]}\left(\xi_{0}\right)$ in (25) , which then reads

$$
\begin{aligned}
\operatorname{Re} \mathcal{F}^{q[\sigma]}(\xi)=\frac{1}{\pi} \int_{1}^{\infty} d \omega \operatorname{Im} C^{q[\sigma]}(\omega) & \int_{0}^{1} d x\left\{F^{q[\sigma]}\left(x, \frac{x}{\omega}\right)\left[\frac{1}{\omega \xi-x}-\sigma \frac{1}{\omega \xi+x}\right]\right. \\
+ & {\left.\left[F^{q[\sigma]}\left(x, \xi_{0}\right)-F^{q[\sigma]}\left(x, \frac{x}{\omega}\right)\right]\left[\frac{1}{\omega \xi_{0}-x}-\sigma \frac{1}{\omega \xi_{0}+x}\right]\right\} . }
\end{aligned}
$$

Notice that the terms in the second line give the amplitude in the limit $\xi \rightarrow \infty$, which corresponds to the point $s=u=q^{2} / 2$ in the unphysical region. The negative-signature amplitude must vanish at this point for symmetry reasons. Comparison of the $\xi_{0}$ independent terms in (24) and (25) shows that an unsubtracted dispersion relation for $\mathcal{F}^{q[-]}(\xi)$ has indeed the same form as (28) without the terms in the second line. The same is however not true for $\mathcal{F}^{q[+]}(\xi)$.

Consistency of the representations (27) and (28) implies

$$
\begin{array}{r}
\frac{1}{\pi} \int_{1}^{\infty} d \omega \operatorname{Im} C^{q[\sigma]}(\omega) \int_{-1}^{1} d x\left[F^{q}(x, \xi)-F^{q}\left(x, \frac{x}{\omega}\right)\right]\left[\frac{1}{\omega \xi-x}-\sigma \frac{1}{\omega \xi+x}\right] \\
=\frac{1}{\pi} \int_{1}^{\infty} d \omega \operatorname{Im} C^{q[\sigma]}(\omega) \int_{-1}^{1} d x\left[F^{q}\left(x, \xi_{0}\right)-F^{q}\left(x, \frac{x}{\omega}\right)\right]\left[\frac{1}{\omega \xi_{0}-x}-\sigma \frac{1}{\omega \xi_{0}+x}\right]
\end{array}
$$

i.e. the 1.h.s. must be independent of $\xi$. In (29) we have restored the integration over negative $x$ and traded $F^{q[\sigma]}$ for $F^{q}$, making use of the symmetry relation (19). The Cauchy principal value prescription should be applied at $x=0$ if $\sigma=-1$, so that a possible nonintegrable singularity of the $\sigma=+1$ part of $F^{q}(x, x / \omega)=\frac{1}{2}\left[F^{q[+]}(x, x / \omega)+F^{q[-]}(x, x / \omega)\right]$ cancels under the integral because it is antisymmetric in $x$. At this point we can make two comments:

1. To leading order in $\alpha_{s}$ the dispersion representation (25) involves only distributions $F^{q[\sigma]}(x, \xi)$ at the point $x=\xi$ because of the simple form (22) of the hard-scattering kernel, as was found in [4]. At higher orders in $\alpha_{s}$ it involves however the distributions in the full DGLAP region $|x| \geq \xi$. Knowledge of $F^{q[\sigma]}(x, x)$ for all $x$ is hence only sufficient to reconstruct the amplitude (up to a subtraction term) at leading order in the strong coupling. The reconstruction is however possible to any order in $\alpha_{s}$ without direct knowledge of the distributions in the ERBL region $|x|<\xi$. 
2. The consistency of dispersion relations for the process amplitude and for the hard-scattering kernel was already discussed in the context of the factorization proof in [3. Translated into our notation, the analog of our eq. (25) in that work was mistakenly written with $F^{q[\sigma]}(x, \xi)$ instead of $F^{q[\sigma]}(x, x / \omega)$ and without a subtraction term, so that consistency with (27) was trivial. The correct consistency relation (29) follows from the polynomiality property of GPDs, as we now show.

\subsection{Consequences for generalized parton distributions}

Clearly (29) is satisfied if

$$
\mathcal{I}^{q[\sigma]}(\omega)=\int_{-1}^{1} d x\left[F^{q}(x, \xi)-F^{q}\left(x, \frac{x}{\omega}\right)\right]\left[\frac{1}{\omega \xi-x}-\sigma \frac{1}{\omega \xi+x}\right]
$$

is independent of $\xi$ for all $\omega \geq 1$. To show that this is the case, we Taylor expand $F^{q}(x, x / \omega)$ in its second argument,

$$
\begin{aligned}
\mathcal{I}^{q[\sigma]}(\omega)= & \left.\frac{1}{\omega} \sum_{n=1}^{\infty} \frac{1}{n !}\left(\frac{\partial}{\partial \eta}\right)^{n} \int_{-1}^{1} d x\left(\frac{x}{\omega}-\xi\right)^{n-1} F^{q}(x, \eta)\right|_{\eta=\xi} \\
& +\left.\sigma \frac{1}{\omega} \sum_{n=1}^{\infty} \frac{1}{n !}\left(\frac{\partial}{\partial \eta}\right)^{n} \int_{-1}^{1} d x\left(\frac{x}{\omega}+\xi\right)^{n-1} F^{q}(x, \eta)\right|_{\eta=-\xi}
\end{aligned}
$$

where we have interchanged the order of differentiation and integration. For definiteness we consider first the case $F^{q}=H^{q}$. Using the polynomiality property (11) and the fact that $C_{n}^{q}$ is only nonzero for even $n$, we find

$$
\mathcal{I}^{q[+]}(\omega)=2 \sum_{\substack{n=2 \\ \text { even }}}^{\infty}\left(\frac{2}{\omega}\right)^{n} C_{n}^{q}, \quad \mathcal{I}^{q[-]}(\omega)=0,
$$

which is independent of $\xi$ as required. We recall that we have suppressed the dependence on $t$ in the distributions $F^{q}$, as well as in the form factors $C_{n}^{q}$. Alternatively one may use the double distribution

representation in (3) and (44). One readily finds that the double distribution part of $\mathcal{I}^{q[\sigma]}$ is zero, with

$$
\begin{aligned}
& \int_{-1}^{1} d x\left[H_{f}^{q}(x, \xi)-H_{f}^{q}\left(x, \frac{x}{\omega}\right)\right] \frac{1}{\omega \xi-x} \\
& \quad=\int_{-1}^{1} d x \int d \beta d \alpha f^{q}(\beta, \alpha)\left[\delta(x-\alpha \xi-\beta)-\delta\left(x\left[1-\frac{\alpha}{\omega}\right]-\beta\right)\right] \frac{1}{\omega \xi-x} \\
& \quad=\int d \beta d \alpha f^{q}(\beta, \alpha)\left[\frac{1}{\omega \xi-\alpha \xi-\beta}-\frac{1 /\left(1-\frac{\alpha}{\omega}\right)}{\omega \xi-\beta /\left(1-\frac{\alpha}{\omega}\right)}\right] \\
& \quad=0
\end{aligned}
$$

and an analogous relation for the term with $1 /(\omega \xi+x)$. The only nonzero contribution to $\mathcal{I}^{q[\sigma]}$ comes hence from the $D$-term

$$
\begin{aligned}
& \mathcal{I}^{q[+]}(\omega)=\operatorname{sign}(\xi) \int_{-1}^{1} d x D^{q}\left(\frac{x}{\xi}\right)\left[\frac{1}{\omega \xi-x}-\frac{1}{\omega \xi+x}\right]=2 \int_{-1}^{1} d \alpha \frac{D^{q}(\alpha)}{\omega-\alpha} \\
& \mathcal{I}^{q[-]}(\omega)=0
\end{aligned}
$$


where we have used the support and symmetry properties of $D^{q}(\alpha)$ stated after (4). Expanding $1 /(\omega-\alpha)$ in a geometric series and using (7) one readily sees that (32) and (34) are equivalent. For the case $F^{q}=E^{q}$ the discussion proceeds in full analogy, with the opposite sign of $C_{n}^{q}$ in (32) and of $D^{q}$ in (34). As a corollary one finds the integral relations

$$
\begin{aligned}
& \int_{-1}^{1} d x\left[H^{q}(x, \xi)-H^{q}\left(x, \frac{x}{\omega}\right)\right]\left[\frac{1}{\omega \xi-x}-\frac{1}{\omega \xi+x}\right] \\
& \quad=-\int_{-1}^{1} d x\left[E^{q}(x, \xi)-E^{q}\left(x, \frac{x}{\omega}\right)\right]\left[\frac{1}{\omega \xi-x}-\frac{1}{\omega \xi+x}\right]=2 \int_{-1}^{1} d x \frac{D^{q}(x)}{\omega-x}
\end{aligned}
$$

and

$$
\begin{aligned}
\int_{-1}^{1} d x & {\left[H^{q}(x, \xi)-H^{q}\left(x, \frac{x}{\omega}\right)\right]\left[\frac{1}{\omega \xi-x}+\frac{1}{\omega \xi+x}\right] } \\
= & \int_{-1}^{1} d x\left[E^{q}(x, \xi)-E^{q}\left(x, \frac{x}{\omega}\right)\right]\left[\frac{1}{\omega \xi-x}+\frac{1}{\omega \xi+x}\right]=0 .
\end{aligned}
$$

They reflect the polynomiality properties of the distributions and in this sense are non-trivial consequences of Lorentz invariance. Using them to evaluate the $\xi_{0}$ dependent terms in (28) gives

$$
\begin{aligned}
\operatorname{Re} \mathcal{H}^{q[+]}(\xi) & =\frac{1}{\pi} \int_{1}^{\infty} d \omega \operatorname{Im} C^{q[+]}(\omega) \int_{-1}^{1} d x\left\{H^{q}\left(x, \frac{x}{\omega}\right)\left[\frac{1}{\omega \xi-x}-\frac{1}{\omega \xi+x}\right]+\frac{2 D^{q}(x)}{\omega-x}\right\} \\
\operatorname{Re} \mathcal{E}^{q[+]}(\xi) & =\frac{1}{\pi} \int_{1}^{\infty} d \omega \operatorname{Im} C^{q[+]}(\omega) \int_{-1}^{1} d x\left\{E^{q}\left(x, \frac{x}{\omega}\right)\left[\frac{1}{\omega \xi-x}-\frac{1}{\omega \xi+x}\right]-\frac{2 D^{q}(x)}{\omega-x}\right\}
\end{aligned}
$$

and

$$
\operatorname{Re} \mathcal{H}^{q[-]}(\xi)=\frac{1}{\pi} \int_{1}^{\infty} d \omega \operatorname{Im} C^{q[-]}(\omega) \int_{-1}^{1} d x H^{q}\left(x, \frac{x}{\omega}\right)\left[\frac{1}{\omega \xi-x}+\frac{1}{\omega \xi+x}\right]
$$

with an analogous representation for $\mathcal{E}^{q[-]}(\xi)$. We note that according to our comment after (28) one has

$$
\lim _{\xi \rightarrow \infty} \mathcal{F}^{q[\sigma]}(\xi)=\frac{1}{\pi} \int_{1}^{\infty} d \omega \operatorname{Im} C^{q[\sigma]}(\omega) \mathcal{I}^{q[\sigma]}(\omega)
$$

For $\sigma=-1$ this is zero, and in fact we could have immediately obtained (38) from an unsubtracted dispersion relation, where the $\xi_{0}$ dependent terms in (28) are absent as remarked earlier. For $\sigma=+1$, the subtraction term in the dispersion relation (25) is fixed by the $D$-term if one takes $\xi_{0} \rightarrow \infty$. In the leading-order approximation for the hard-scattering kernel this was already observed in [4, and for the general case in [1]. According to Table 1 the $D$-term parameterizes a part of $H^{q}$ and $E^{q}$ which is associated with spin-zero exchange in the $t$-channel 3 From (17) one readily finds that its contribution to the invariant amplitudes $\mathcal{H}^{q[+]}(\xi)$ and $\mathcal{E}^{q[+]}(\xi)$ is energy-independent and purely real.

\footnotetext{
${ }^{3}$ Note that this is not restricted to the exchange of spin-zero resonances. In the context of chiral dynamics 20, the dominant exchange is in fact given by two pions in an $S$-wave.
} 


\subsection{The Compton amplitude with both photons off shell}

So far we have discussed deeply virtual Compton scattering, $\gamma^{*} p \rightarrow \gamma p$, where the photon in the final state is on shell, and obtained the integral relations (35) and (36) for the generalized parton distributions. It is natural to ask whether any further relations can be derived by considering dispersion relations for the Compton amplitude

$$
\gamma^{*}(q)+p(p) \rightarrow \gamma^{*}\left(q^{\prime}\right)+p\left(p^{\prime}\right)
$$

with both photons off shell. For $q^{2}<0$ and $q^{2}>0$ this process can be studied experimentally, with the timelike final-state photon decaying into a lepton pair [21]. The analyticity properties of the amplitude are however more complicated in this case, because there are simultaneous branch cuts in $s$ and $q^{\prime 2}$ or in $u$ and $q^{\prime 2}$. Instead we consider the case where both $q^{2}$ and $q^{\prime 2}$ are spacelike, so that the only singularities are in $s$ and $u$, as in the previous subsections. We have two scaling variables

$$
\xi=-\frac{\left(q+q^{\prime}\right)^{2}}{2\left(p+p^{\prime}\right) \cdot\left(q+q^{\prime}\right)}=-\frac{q^{2}+q^{\prime 2}}{s-u}, \quad \vartheta=\frac{q^{2}-q^{2}}{q^{2}+q^{\prime 2}}
$$

where in the second expression for $\xi$ we have neglected $t$ compared with $q^{2}+q^{\prime 2}$. For $\vartheta=1$ we recover the case of DVCS, whereas with two spacelike photon virtualities we have $-1<\vartheta<1$. In the Bjorken limit of large $-q^{2}$ at fixed $\xi, \vartheta$ and $t$ one has a factorization formula for the invariant amplitudes

$$
\mathcal{F}^{q[\sigma]}\left(\xi, \vartheta, t, q^{2}\right)=\int_{-1}^{1} d x \frac{1}{\xi} C^{q[\sigma]}\left(\frac{x}{\xi}, \vartheta, q^{2}\right) F^{q}(x, \vartheta \xi, t)
$$

with $F^{q}=H^{q}, E^{q}$ as before. We will again omit the arguments $q^{2}$ and $t$ in the following. The Mandelstam variables of the hard subprocess now read

$$
\hat{s}=x s+\frac{1}{2}(1-x)\left(q^{2}+q^{2}\right), \quad \hat{u}=x u+\frac{1}{2}(1-x)\left(q^{2}+q^{\prime 2}\right),
$$

in the Bjorken limit, so that $x / \xi=-(\hat{s}-\hat{u}) /\left(q^{2}+q^{\prime 2}\right)$. For a dispersion relation at fixed $t$ and fixed photon virtualities, $\vartheta$ plays the role of a constant parameter, and we can use $1 / \xi$ and $x / \xi$ as respective energy variable of the overall process and the hard subprocess. In the Bjorken limit the corresponding amplitudes have branch cuts in $1 / \xi$ or $x / \xi$ from 1 to $\infty$ and from $-\infty$ to -1 . The hard-scattering kernel has the symmetry

$$
C^{q[\sigma]}\left(-\frac{x}{\xi}, \vartheta\right)=-\sigma C^{q[\sigma]}\left(\frac{x}{\xi}, \vartheta\right)
$$

in analogy to (16). At leading order in $\alpha_{s}$ it reads

$$
C^{q[\sigma]}(\omega, \vartheta) \propto \frac{1}{1-\omega-i \epsilon}-\sigma \frac{1}{1+\omega-i \epsilon}, \quad \operatorname{Im} C^{q[\sigma]}(\omega, \vartheta) \propto \pi[\delta(\omega-1)-\sigma \delta(\omega+1)],
$$

and at higher orders it has the same high- $\omega$ behavior as discussed for DVCS after (22). In other words, the high-energy behavior of the hard-scattering kernel for the virtual Compton amplitude (40) remains unchanged if $q^{\prime 2} \rightarrow 0$. Similarly, the small- $\xi$ behavior of $\mathcal{F}^{q[\sigma]}(\xi, \vartheta)$ is as discussed for DVCS after (23). One can thus derive dispersion relations for the invariant amplitude and for the hard-scattering kernel as in Sect. 3.1 and finds

$$
\operatorname{Re} \mathcal{F}^{q[\sigma]}(\xi, \vartheta)=\frac{1}{\pi} \int_{1}^{\infty} d \omega \operatorname{Im} C^{q[\sigma]}(\omega, \vartheta) \int_{0}^{1} d x F^{q[\sigma]}(x, \vartheta \xi)\left[\frac{1}{\omega \xi-x}-\sigma \frac{1}{\omega \xi+x}\right]
$$


and

$$
\begin{aligned}
\operatorname{Re} \mathcal{F}^{q[\sigma]}(\xi, \vartheta)=\frac{1}{\pi} \int_{1}^{\infty} d \omega & \operatorname{Im} C^{q[\sigma]}(\omega, \vartheta) \int_{0}^{1} d x\left\{F^{q[\sigma]}\left(x, \vartheta \frac{x}{\omega}\right)\left[\frac{1}{\omega \xi-x}-\sigma \frac{1}{\omega \xi+x}\right]\right. \\
+ & {\left.\left[F^{q[\sigma]}\left(x, \vartheta \xi_{0}\right)-F^{q[\sigma]}\left(x, \vartheta \frac{x}{\omega}\right)\right]\left[\frac{1}{\omega \xi_{0}-x}-\sigma \frac{1}{\omega \xi_{0}+x}\right]\right\} . }
\end{aligned}
$$

These relations read exactly as their counterparts (27) and (28) for DVCS, except that the second argument of $F^{q[\sigma]}$ is now multiplied with $\vartheta$ and that $C^{q[\sigma]}$ depends on $\vartheta$ as well. The consistency of (46) and (47) is ensured if

$$
\int_{-1}^{1} d x\left[F^{q}(x, \vartheta \xi)-F^{q}\left(x, \vartheta \frac{x}{\omega}\right)\right]\left[\frac{1}{\omega \xi-x}-\sigma \frac{1}{\omega \xi+x}\right]
$$

is independent of $\xi$ for all $\omega \geq 1$. Rescaling $\xi^{\prime}=\vartheta \xi$ and $\omega^{\prime}=\omega / \vartheta$, we readily see that this in ensured by the $\xi$-independence of the integral $\mathcal{I}^{q[\sigma]}(\omega)$ in $(30)$, which we have already established. Thus the dispersion relations for doubly virtual Compton scattering give no new relations for GPDs. Of course, one obtains dispersion representations for $\mathcal{H}^{q[\sigma]}(\xi, \vartheta)$ and $\mathcal{E}^{q[\sigma]}(\xi, \vartheta)$ as in (37) and (38), with $\vartheta$ as an additional argument in $C^{q[\sigma]}$ and with the replacements $H^{q}(x, x / \omega) \rightarrow H^{q}(x, \vartheta x / \omega)$, $E^{q}(x, x / \omega) \rightarrow E^{q}(x, \vartheta x / \omega)$ and $D^{q}(x)(\omega-x)^{-1} \rightarrow D^{q}(x)(\omega / \vartheta-x)^{-1}$.

Let us now consider the case $q=q^{\prime}$, relevant for deep inelastic scattering, where we have $\xi=x_{B}$ and $\vartheta=0$. The representations (46) and (47) are then trivially consistent, because the second argument of $F^{q[\sigma]}$ is zero everywhere. In other words, the usual parton densities appearing in inclusive processes do not depend on an external kinematical variable, unlike the generalized parton distributions appearing in exclusive processes.

In the following section we investigate the contributions from polarized quark distributions and from gluons to DVCS and to meson production. The results we will obtain can readily be generalized to the Compton amplitude with two spacelike photons. We note that for the unpolarized quark distributions we have just considered, only the amplitudes with $\sigma=+1$ appear in Compton scattering, whereas $\sigma=-1$ is relevant for the polarized quark and gluon distributions.

\section{Polarized and gluon distributions}

Contributions from polarized quarks and from unpolarized or polarized gluons to invariant amplitudes can be treated in a similar manner as the case of unpolarized quarks in the previous section. Particularities arise for each of the distributions, which we will now discuss in turn.

\subsection{Polarized quark distributions}

Let us first investigate invariant amplitudes involving polarized quark distributions, which appear in both DVCS and in the production of pseudoscalar mesons. The factorization formula reads as in (15), where now $F^{q}=\widetilde{H}^{q}$ or $\widetilde{E}^{q}$. We define combinations $\widetilde{H}^{q[\sigma]}$ and $\widetilde{E}^{q[\sigma]}$ of definite signature as in (18), and the relations (16) to (19) are again valid. Note that, in contrast to their unpolarized counterparts, $\widetilde{H}^{q[+]}$ and $\widetilde{E}^{q[+]}$ correspond to negative charge conjugation and $\widetilde{H}^{q[-]}$ and $\widetilde{E}^{q[-]}$ to positive charge conjugation in the $t$-channel. The leading-order expression of the hard-scattering kernel for DVCS and for meson production is the same as in (22). At NLO one finds a large- $\omega$ behavior $C^{q[\sigma]}(\omega) \sim \omega^{-1}$ 
up to logarithms in both cases. Note that $t$-channel two-gluon exchange in the polarized sector does not give rise to a power behavior as $\omega^{0}$. This is also explicitly seen in the NNLO kernels for inclusive deep inelastic scattering [22].

For the polarized quark and antiquark densities we assume that $x \Delta q(x)$ and $x \Delta \bar{q}(x)$ vanish at $x \rightarrow 0$, as it is found in global fits and required for the existence of the moments $\int_{0}^{1} d x \Delta q(x)$ and $\int_{0}^{1} d x \Delta \bar{q}(x)$. One should then have a small- $\xi$ behavior $\xi \widetilde{\mathcal{H}}^{q[\sigma]} \rightarrow 0$ for both positive and negative signature, so that the once-subtracted dispersion relation (23) is valid. The argument proceeds as in Sections 3.1 and 3.2. According to (1) the $x^{n-1}$ moment of $\widetilde{H}^{q}(x, \xi)$ has $\xi^{n-1}$ as highest power, so that the integral $\mathcal{I}^{[\sigma]}(\omega)$ in (30) is zero for both $\sigma=+1$ and $\sigma=-1$ in this case. We therefore obtain the integral relation

$$
\int_{-1}^{1} d x\left[\widetilde{H}^{q}(x, \xi)-\widetilde{H}^{q}\left(x, \frac{x}{\omega}\right)\right]\left[\frac{1}{\omega \xi-x} \pm \frac{1}{\omega \xi+x}\right]=0
$$

and dispersion representations

$$
\begin{aligned}
\operatorname{Re} \widetilde{\mathcal{H}}^{q[\sigma]}(\xi) & =\frac{1}{\pi} \int_{1}^{\infty} d \omega \operatorname{Im} C^{q[\sigma]}(\omega) \int_{-1}^{1} d x \widetilde{H}^{q}(x, \xi)\left[\frac{1}{\omega \xi-x}-\sigma \frac{1}{\omega \xi+x}\right] \\
& =\frac{1}{\pi} \int_{1}^{\infty} d \omega \operatorname{Im} C^{q[\sigma]}(\omega) \int_{-1}^{1} d x \widetilde{H}^{q}\left(x, \frac{x}{\omega}\right)\left[\frac{1}{\omega \xi-x}-\sigma \frac{1}{\omega \xi+x}\right] .
\end{aligned}
$$

We further find that $\widetilde{\mathcal{H}}^{q[ \pm]}(\xi) \rightarrow 0$ for $\xi \rightarrow \infty$. As in the unpolarized case, we could have obtained the second representation in (50) from a dispersion relation without subtraction in the case $\sigma=-1$. For $\sigma=+1$, the high-energy behavior of the invariant amplitude does however require one subtraction, even though the subtraction term is zero when taking the subtraction point $\xi_{0} \rightarrow \infty$. An unsubtracted dispersion relation for positive signature would differ from (50), as remarked after (28).

For $\widetilde{E}^{q[\sigma]}$ the situation is more involved. According to Table 2 this distribution admits more $t$ channel exchanges than $\widetilde{H}^{q[\sigma]}$, so that the small- $\xi$ behavior of $\widetilde{\mathcal{E}}^{q[\sigma]}(\xi)$ and $\widetilde{\mathcal{H}}^{q[\sigma]}(\xi)$ may be different. In particular there is a known spin-zero exchange contribution to $\widetilde{E}^{q[-]}$, which is due to pion exchange and dominates the distributions for $u$ and $d$ quarks at small $t$ [23, 24]. It reads

$$
\widetilde{E}_{\pi}^{u}(x, \xi, t)=-\widetilde{E}_{\pi}^{d}(x, \xi, t)=\frac{c}{m_{\pi}^{2}-t} \frac{1}{|\xi|} \phi_{\pi}\left(\frac{x}{\xi}\right)
$$

where the constant $c$ can be calculated in chiral perturbation theory [20] and the light-cone distribution amplitude $\phi_{\pi}(\alpha)$ of the pion is an even function with support for $|\alpha|<1$. Inserting this into the factorization formula (15) one obtains a contribution going like $\xi^{-1}$ to the invariant amplitudes $\widetilde{\mathcal{E}}^{u[-]}$ and $\widetilde{\mathcal{E}}^{d[-]}$. This rises too strongly at $\xi \rightarrow 0$ for the once-subtracted dispersion relations we have used so far. At this point we notice that due to the prefactor in its definition, the distribution $\widetilde{E}^{q}$ always contributes to matrix elements as $\xi \widetilde{E}^{q}$, and correspondingly it is $\xi \widetilde{\mathcal{E}}^{q[\sigma]}$ which appears in physical scattering amplitudes. Note that because of its prefactor $\xi \widetilde{\mathcal{E}}^{q[-]}(\xi)$ is even in $\xi$ and thus has positive instead of negative signature. The pion exchange term (51) gives a $\xi$ independent contribution to $\xi \widetilde{\mathcal{E}}^{q[-]}(\xi)$, as it should be for spin-zero exchange. We can thus write down a once-subtracted dispersion relation for $\xi \widetilde{\mathcal{E}}^{q[\sigma]}(\xi)$, assuming only that its small- $\xi$ behavior is less singular than $\xi^{-2}$ for $\sigma=-1$ and less singular than $\xi^{-1}$ for $\sigma=+1$. The analog of (27) is now

$$
\operatorname{Re} \xi \widetilde{\mathcal{E}}^{q[\sigma]}(\xi)=\frac{1}{\pi} \int_{1}^{\infty} d \omega \operatorname{Im} C^{q[\sigma]}(\omega) \int_{-1}^{1} d x \xi \widetilde{E}^{q}(x, \xi)\left[\frac{1}{\omega \xi-x}-\sigma \frac{1}{\omega \xi+x}\right]
$$


and the analog of (28) reads

$$
\begin{aligned}
\operatorname{Re} \xi \widetilde{\mathcal{E}}^{q[\sigma]}(\xi)=\frac{1}{\pi} \int_{1}^{\infty} & d \omega \operatorname{Im} C^{q[\sigma]}(\omega) \int_{-1}^{1} d x\left\{\xi_{0} \widetilde{E}^{q}\left(x, \xi_{0}\right)\left[\frac{1}{\omega \xi_{0}-x}-\sigma \frac{1}{\omega \xi_{0}+x}\right]\right. \\
& \left.+\frac{x}{\omega} \widetilde{E}^{q}\left(x, \frac{x}{\omega}\right)\left[\frac{1}{\omega \xi-x}+\sigma \frac{1}{\omega \xi+x}-\frac{1}{\omega \xi_{0}-x}-\sigma \frac{1}{\omega \xi_{0}+x}\right]\right\},
\end{aligned}
$$

which can be rewritten as

$$
\begin{aligned}
\operatorname{Re} \xi \widetilde{\mathcal{E}}^{q[\sigma]}(\xi)=\frac{1}{\pi} \int_{1}^{\infty} d \omega \operatorname{Im} C^{q[\sigma]}(\omega) & \int_{-1}^{1} d x\left\{\xi \widetilde{E}^{q}\left(x, \frac{x}{\omega}\right)\left[\frac{1}{\omega \xi-x}-\sigma \frac{1}{\omega \xi+x}\right]\right. \\
& \left.+\xi_{0}\left[\widetilde{E}^{q}\left(x, \xi_{0}\right)-\widetilde{E}^{q}\left(x, \frac{x}{\omega}\right)\right]\left[\frac{1}{\omega \xi_{0}-x}-\sigma \frac{1}{\omega \xi_{0}+x}\right]\right\} .
\end{aligned}
$$

With the methods of Sect. 3.2 one finds

$$
\int_{-1}^{1} d x\left[\widetilde{E}^{q}(x, \xi)-\widetilde{E}^{q}\left(x, \frac{x}{\omega}\right)\right]\left[\frac{1}{\omega \xi-x} \pm \frac{1}{\omega \xi+x}\right]=0,
$$

which ensures consistency of the two dispersion representations and allows us to omit the second line of (54). We thus find that the analog of the representations (501) also holds for $\widetilde{\mathcal{E}}^{q[\sigma]}$. In the case $\sigma=+1$, where spin-zero exchange does not contribute, we could indeed have obtained this result from a once-subtracted dispersion relation for $\widetilde{\mathcal{E}}^{q[\sigma]}$.

Notice that the terms in the second line of (54) need not give $\xi \widetilde{\mathcal{E}}^{q[\sigma]}(\xi)$ at the unphysical point $\xi \rightarrow \infty$, in contrast to the case discussed after (28). In fact $\xi \widetilde{\mathcal{E}}^{q[-]}(\xi)$ is nonzero at this point. Taylor expanding $1 /(\omega \xi-x)$ and $1 /(\omega \xi+x)$ in (52) and using the polynomiality relation (1) one readily finds

$$
\lim _{\xi \rightarrow \infty} \xi \widetilde{\mathcal{E}}^{q[-]}(\xi)=\frac{1}{\pi} \int_{1}^{\infty} d \omega \operatorname{Im} C^{q[-]}(\omega) \sum_{\substack{n=1 \\ \text { odd }}}^{\infty}\left(\frac{2}{\omega}\right)^{n} \widetilde{B}_{n, n-1}^{q}, \quad \lim _{\xi \rightarrow \infty} \xi \widetilde{\mathcal{E}}^{q[+]}(\xi)=0 .
$$

In Table 1 we see that the form factors $\widetilde{B}_{n, n-1}^{q}(t)$ are associated with pure spin-zero exchange. At small $t$ they are dominated by the the pion-exchange term (51). Having support only in the ERBL region $|x|<\xi$, this term does not contribute to the imaginary part of $\xi_{\mathcal{E}^{q[}}{ }^{[-]}(\xi)$, and one may wonder how it can appear in the representation (54) for the real part. The answer is that it induces a contribution proportional to $\delta(x)$ in $\widetilde{E}^{q}(x, x / \omega)$. To see this we observe that the double distribution generating (51) has the form $\delta(\beta) e_{\pi}(\alpha, t)$, where we have abbreviated $e_{\pi}(\alpha, t)=c\left(m_{\pi}^{2}-t\right)^{-1} \phi_{\pi}(\alpha)$. For $\omega \geq 1$ one then has

$$
\widetilde{E}_{\pi}^{u}\left(x, \frac{x}{\omega}, t\right)=\int d \beta d \alpha \delta\left(x\left[1-\frac{\alpha}{\omega}\right]-\beta\right) \delta(\beta) e_{\pi}(\alpha, t)=\delta(x) \omega \int_{-1}^{1} d \alpha \frac{e_{\pi}(\alpha, t)}{\omega-\alpha} .
$$

One may avoid this $\delta(x)$ contribution by taking the limit $\xi_{0} \rightarrow \infty$ in (53), which yields

$$
\begin{aligned}
\operatorname{Re} \xi \widetilde{\mathcal{E}}^{q[\sigma]}(\xi)= & \lim _{\xi_{0} \rightarrow \infty} \xi_{0} \widetilde{\mathcal{E}}^{q[\sigma]}\left(\xi_{0}\right) \\
& +\frac{1}{\pi} \int_{1}^{\infty} d \omega \operatorname{Im} C^{q[\sigma]}(\omega) \int_{-1}^{1} d x \frac{x}{\omega} \widetilde{E}^{q}\left(x, \frac{x}{\omega}\right)\left[\frac{1}{\omega \xi-x}+\sigma \frac{1}{\omega \xi+x}\right]
\end{aligned}
$$

with the subtraction term given in (56). The $\delta(x)$ contribution in $\widetilde{E}^{q}(x, x / \omega)$ is now removed by the extra factor $x$ and instead appears explicitly in the subtraction term. 


\subsection{Unpolarized gluon distributions}

The contribution from unpolarized gluon distributions to invariant amplitudes can be written as

$$
\mathcal{F}^{g}(\xi)=\int_{-1}^{1} d x \frac{1}{\xi} C^{g}\left(\frac{x}{\xi}\right) \frac{F^{g}(x, \xi)}{x},
$$

where $F^{g}(x, \xi)=H^{g}(x, \xi), E^{g}(x, \xi)$ is even in $x$ and in $\xi$, and the hard-scattering kernel $C^{g}(\omega)$ is odd in $\omega$. The singularity introduced by the factor $1 / x$ is spurious because $C^{g}(\omega) \sim \omega$ at $\omega \rightarrow 0$. For vector meson production, the hard-scattering kernel reads

$$
C^{g}(\omega) \propto \frac{1}{1-\omega-i \epsilon}-\frac{1}{1+\omega-i \epsilon}, \quad \quad \operatorname{Im} C^{g}(\omega) \propto \pi[\delta(\omega-1)-\delta(\omega+1)]
$$

at LO in $\alpha_{s}$, whereas for DVCS the kernel for gluon distributions starts only at NLO. The high- $\omega$ behavior of $C^{g}(\omega)$ at higher orders is the same as discussed for $C^{q[+]}(\omega)$ after (22). We assume a small- $x$ behavior like $g(x) \sim x^{-\alpha}$ with $\alpha<2$ for the unpolarized gluon density. The small- $\xi$ behavior $\mathcal{H}^{g}(\xi) \sim \xi^{-\alpha}$ is then less singular than $\xi^{-2}$ and hence admits a once-subtracted dispersion relation.

The symmetry properties of $C^{g}(\omega)$ and of $x^{-1} F^{g}(x, \xi)$ are identical to those of $C^{q[+]}(\omega)$ and $F^{q[+]}(x, \xi)$ in the unpolarized quark sector, so that the dispersion relations for the process amplitude and for the hard-scattering kernel read exactly as for unpolarized quark distributions in (27) and (28) if one replaces $\mathcal{F}^{q[+]} \rightarrow \mathcal{F}^{g}, C^{q[+]} \rightarrow C^{g}$ and $F^{q[+]} \rightarrow 2 x^{-1} F^{g}$. Consistency of these dispersion relations is ensured if

$$
\mathcal{I}^{g}(\omega)=\int_{-1}^{1} \frac{d x}{x}\left[F^{g}(x, \xi)-F^{g}\left(x, \frac{x}{\omega}\right)\right]\left[\frac{1}{\omega \xi-x}-\frac{1}{\omega \xi+x}\right]
$$

is independent of $\xi$. Using the symmetry properties of $F^{g}$ we can replace $1 /(\omega \xi-x)-1 /(\omega \xi+x)$ by $2 /(\omega \xi-x)$ under the integral, with the principal value prescription taken to regularize the singularity at $x=0$. Repeating the procedure of Section 3.2 we Taylor expand $F^{g}(x, x / \omega)$ in its second argument and obtain

$$
\mathcal{I}^{g}(\omega)=\left.\frac{2}{\omega} \sum_{n=1}^{\infty} \frac{1}{n !}\left(\frac{\partial}{\partial \eta}\right)^{n} \int_{-1}^{1} \frac{d x}{x}\left(\frac{x}{\omega}-\xi\right)^{n-1} F^{g}(x, \eta)\right|_{\eta=\xi}
$$

Since $F^{g}(x, \eta)$ is even in $x$, a nonzero integral is only obtained from the odd powers of $x$ in the expansion of $(x / \omega-\xi)^{n-1}$, so that the factor $x^{-1}$ in the integrand is canceled. Using the polynomial property (2) one finally obtains

$$
\mathcal{I}^{g}(\omega)=4 \sum_{\substack{n=2 \\ \text { even }}}^{\infty}\left(\frac{2}{\omega}\right)^{n} C_{n}^{g}
$$

for $F^{g}=H^{g}$, which is independent of $\xi$ as required. Alternatively, one may insert (5) and (6) into (61). For the double distribution part of $H^{g}$ this gives

$$
\begin{aligned}
\int_{-1}^{1} & \frac{d x}{x}\left[H_{f}^{g}(x, \xi)-H_{f}^{g}\left(x, \frac{x}{\omega}\right)\right]\left[\frac{1}{\omega \xi-x}-\frac{1}{\omega \xi+x}\right] \\
& =2 \int_{-1}^{1} d x \int d \beta d \alpha \beta f^{g}(\beta, \alpha)\left[\delta(x-\alpha \xi-\beta)-\delta\left(x\left[1-\frac{\alpha}{\omega}\right]-\beta\right)\right] \frac{1}{x(\omega \xi-x)} \\
& =-\frac{2}{\omega} \int d \beta d \alpha \frac{\alpha f^{g}(\beta, \alpha)}{\alpha \xi+\beta}
\end{aligned}
$$


which is zero because $\alpha f^{g}(\beta, \alpha)$ is odd in both $\beta$ and in $\alpha$. The $D$-term contribution to $H^{g}$ gives

$$
\mathcal{I}^{g}(\omega)=\frac{2}{\omega} \int_{-1}^{1} d \alpha \frac{D^{g}(\alpha)}{\omega-\alpha}
$$

in agreement with (7) and (63) . For $F^{g}=E^{g}$ one finds analogous results with the opposite sign for $C^{g}$ and $D^{g}$. One thus obtains integral relations

$$
\begin{aligned}
& \int_{-1}^{1} \frac{d x}{x}\left[H^{g}(x, \xi)-H^{g}\left(x, \frac{x}{\omega}\right)\right]\left[\frac{1}{\omega \xi-x}-\frac{1}{\omega \xi+x}\right] \\
& =-\int_{-1}^{1} \frac{d x}{x}\left[E^{g}(x, \xi)-E^{g}\left(x, \frac{x}{\omega}\right)\right]\left[\frac{1}{\omega \xi-x}-\frac{1}{\omega \xi+x}\right]=\frac{2}{\omega} \int_{-1}^{1} d x \frac{D^{g}(x)}{\omega-x}
\end{aligned}
$$

and dispersion representations

$$
\begin{aligned}
& \operatorname{Re} \mathcal{H}^{g}(\xi)=\frac{1}{\pi} \int_{1}^{\infty} d \omega \operatorname{Im} C^{g}(\omega) \int_{-1}^{1} d x\left\{\frac{1}{x} H^{g}\left(x, \frac{x}{\omega}\right)\left[\frac{1}{\omega \xi-x}-\frac{1}{\omega \xi+x}\right]+\frac{2}{\omega} \frac{D^{g}(x)}{\omega-x}\right\}, \\
& \operatorname{Re} \mathcal{E}^{g}(\xi)=\frac{1}{\pi} \int_{1}^{\infty} d \omega \operatorname{Im} C^{g}(\omega) \int_{-1}^{1} d x\left\{\frac{1}{x} E^{g}\left(x, \frac{x}{\omega}\right)\left[\frac{1}{\omega \xi-x}-\frac{1}{\omega \xi+x}\right]-\frac{2}{\omega} \frac{D^{g}(x)}{\omega-x}\right\} .
\end{aligned}
$$

Furthermore one finds

$$
\lim _{\xi \rightarrow \infty} \mathcal{H}^{g}(\xi)=-\lim _{\xi \rightarrow \infty} \mathcal{E}^{g}(\xi)=\frac{1}{\pi} \int_{1}^{\infty} \frac{d \omega}{\omega} \operatorname{Im} C^{g}(\omega) \int_{-1}^{1} d x \frac{2 D^{g}(x)}{\omega-x}
$$

for the invariant amplitudes at $\xi \rightarrow \infty$. We remark in passing that (66) and (67) may be rewritten using

$$
\frac{1}{x}\left[\frac{1}{\omega \xi-x}-\frac{1}{\omega \xi+x}\right]=\frac{1}{\omega \xi}\left[\frac{1}{\omega \xi-x}+\frac{1}{\omega \xi+x}\right] \text {. }
$$

\subsection{Polarized gluon distributions}

Let us now discuss the generalized gluon distributions in the polarized sector, which appear in DVCS starting at NLO in $\alpha_{s}$. As in the previous section we begin with the factorization formula (59), where now $F^{g}(x, \xi)=\widetilde{H}^{g}(x, \xi), \widetilde{E}^{g}(x, \xi)$ is odd in $x$. The hard-scattering kernel $C^{g}$ is even in $\omega$ and vanishes like $\omega^{2}$ for $\omega \rightarrow 0$. The invariant amplitudes $\widetilde{\mathcal{H}}^{g}(\xi)$ and $\widetilde{\mathcal{E}}^{g}(\xi)$ have negative signature. The NLO calculation of $C^{g}(\omega)$ for DVCS gives a large- $\omega$ behavior like $\omega^{-1}$ up to logarithms, and higher orders will have the same power behavior as discussed in the first paragraph of Sect. 4.1,

Assuming a small- $x$ behavior $x \Delta g(x) \rightarrow 0$ of the polarized gluon density, which is required for the existence of the moment $\int_{0}^{1} d x \Delta g(x)$ and consistent with global fits of parton densities, we expect that $\xi \widetilde{\mathcal{H}}^{g}(\xi) \rightarrow 0$ for $\xi \rightarrow 0$. We then readily obtain dispersion relations as in (27) and (28) with the replacements $\mathcal{F}^{q[-]} \rightarrow \widetilde{\mathcal{H}}^{g}, C^{q[-]} \rightarrow C^{g}$ and $F^{q[-]} \rightarrow 2 x^{-1} \widetilde{H}^{g}$. Their consistency requires the $\xi$-independence of

$$
\mathcal{I}^{g}(\omega)=\int_{-1}^{1} \frac{d x}{x}\left[\widetilde{H}^{g}(x, \xi)-\widetilde{H}^{g}\left(x, \frac{x}{\omega}\right)\right]\left[\frac{1}{\omega \xi-x}+\frac{1}{\omega \xi+x}\right]
$$


where the principal value prescription is to be taken at $x=0$. As in Sect. 4.2 we can rewrite this as

$$
\begin{aligned}
\mathcal{I}^{g}(\omega) & =\left.\frac{2}{\omega} \sum_{n=1}^{\infty} \frac{1}{n !}\left(\frac{\partial}{\partial \eta}\right)^{n} \int_{-1}^{1} \frac{d x}{x}\left(\frac{x}{\omega}-\xi\right)^{n-1} \widetilde{H}^{g}(x, \eta)\right|_{\eta=\xi} \\
& =\left.\frac{2}{\omega} \sum_{n=1}^{\infty} \frac{1}{n !}(-\xi)^{n-1}\left(\frac{\partial}{\partial \eta}\right)^{n} \int_{-1}^{1} \frac{d x}{x} \widetilde{H}^{g}(x, \eta)\right|_{\eta=\xi},
\end{aligned}
$$

where in the second step we have expanded the factor $(x / \omega-\xi)^{n-1}$ and used the polynomiality properties (2) of $\widetilde{H}^{g}$. To proceed we need to know the dependence of $\int d x x^{-1} \widetilde{H}^{g}(x, \eta)$ on $\eta$.

In [7] a double distribution representation for $\widetilde{H}^{g}$ was given, which has the same form as (6) for $H_{f}^{g}$. Inserting this into (70) one obtains an expression as in (64), which is nonzero because the corresponding double distribution is even and not odd in $\beta$. Such a double distribution representation for $\widetilde{H}^{g}$ (as well as its analog for $\widetilde{E}^{g}$ ) is however incomplete, because for the $x^{n-2}$ moment of the distributions it gives a polynomial with highest power $\xi^{n-3}$ (with $n$ being odd) instead of $\xi^{n-1}$ as required in (2). To obtain a correct representation, we can use the construction discussed in [25] for the generalized quark distribution in the pion. This leads to writing a double distribution representation for $x^{-1} \widetilde{H}^{g}$ and $x^{-1} \widetilde{E}^{g}$, i.e.

$$
\begin{aligned}
\widetilde{H}^{g}(x, \xi, t) & =x \int d \beta d \alpha \delta(x-\alpha \xi-\beta) \widetilde{f}^{g}(\beta, \alpha, t), \\
\widetilde{E}^{g}(x, \xi, t) & =x \int d \beta d \alpha \delta(x-\alpha \xi-\beta) \widetilde{k}^{g}(\beta, \alpha, t),
\end{aligned}
$$

where $\widetilde{f}^{g}$ and $\widetilde{k}^{g}$ are even in $\alpha$ and $\beta$. We note that in the forward limit $t=0$ one has $\int d \alpha \widetilde{f}^{g}(x, \alpha, 0)=$ $\Delta g(x)$, which is much less singular than the corresponding limit $x^{-1} q_{\pi}(x)$ for the double distribution of quarks in the pion considered in [25] and should thus be less problematic for the purpose of model building.

Apart from giving the required maximum power of $\xi^{n-1}$ for the $x^{n-2}$ moments of $\widetilde{H}^{g}$ and $\widetilde{E}^{g}$, the representation (72) also has the important consequence that

$$
\int_{-1}^{1} \frac{d x}{x} \widetilde{H}^{g}(x, \eta)=\int d \beta d \alpha \widetilde{f}^{g}(\beta, \alpha)
$$

is independent of $\eta$, so that according to (71)

$$
\mathcal{I}^{g}(\omega)=0
$$

is independent of $\xi$, which we had to show. This is also seen by direct insertion of (72) into (70), which leads to an expression of the form (33) we encountered for quark distributions. We thus finally obtain dispersion representations as in (50) with the replacements $\widetilde{\mathcal{H}}^{q[-]} \rightarrow \widetilde{\mathcal{H}}^{g}, C^{q[-]} \rightarrow C^{g}$ and $\widetilde{H}^{q} \rightarrow x^{-1} \widetilde{H}^{g}$, as well as the limit $\widetilde{\mathcal{H}}^{g}(\xi) \rightarrow 0$ for $\xi \rightarrow \infty$.

For the invariant amplitude $\widetilde{\mathcal{E}}^{g}$ we must take into account a possible spin-zero exchange in the $t$-channel (although the exchange of an $\eta$ or $\eta^{\prime}$ in the flavor singlet sector is most likely not of the same phenomenological importance as pion exchange in $\widetilde{E}^{q}$ ). With the double distribution representation (72) one can proceed exactly as for the case of quark distributions in Sect. 4.1. One thus obtains analogs of the dispersion representations (150) with the replacements $\widetilde{\mathcal{H}}^{q[-]} \rightarrow \widetilde{\mathcal{E}}^{g}, C^{q[-]} \rightarrow C^{g}$ and $\widetilde{H}^{q} \rightarrow x^{-1} \widetilde{E}^{g}$, as well as the results

$$
\int_{-1}^{1} \frac{d x}{x}\left[\widetilde{E}^{g}(x, \xi)-\widetilde{E}^{g}\left(x, \frac{x}{\omega}\right)\right]\left[\frac{1}{\omega \xi-x}+\frac{1}{\omega \xi+x}\right]=0
$$


and

$$
\lim _{\xi \rightarrow \infty} \xi \widetilde{\mathcal{E}}^{g}(\xi)=\frac{1}{\pi} \int_{1}^{\infty} d \omega \operatorname{Im} C^{g}(\omega) \sum_{\substack{n=1 \\ \text { odd }}}^{\infty}\left(\frac{2}{\omega}\right)^{n} 2 \widetilde{B}_{n, n-1}^{g} .
$$

To avoid a $\delta(x)$ contribution in $x^{-1} \widetilde{E}^{g}(x, x / \omega)$ due to spin-zero exchange one may use the analog of (58), which reads

$$
\operatorname{Re} \xi \widetilde{\mathcal{E}}^{g}(\xi)=\lim _{\xi_{0} \rightarrow \infty} \xi_{0} \widetilde{\mathcal{E}}^{g}\left(\xi_{0}\right)+\frac{1}{\pi} \int_{1}^{\infty} \frac{d \omega}{\omega} \operatorname{Im} C^{g}(\omega) \int_{-1}^{1} d x \widetilde{E}^{g}\left(x, \frac{x}{\omega}\right)\left[\frac{1}{\omega \xi-x}-\frac{1}{\omega \xi+x}\right] .
$$

\subsection{Helicity-flip distributions}

We conclude this section with a few remarks on the generalized parton distributions for quark or gluon helicity flip, which have been introduced and discussed in [26, 27].

In the quark case these distributions are chiral-odd, and to date there is no simple exclusive process known where they appear. Reactions like $\gamma^{*} p \rightarrow \rho \rho p$ were proposed in [28, but due to their three-particle final state the discussion of dispersion relations would be much more complicated. However, integral relations analogous to (49) are valid for the quark distributions $H_{T}^{q}, E_{T}^{q}, \widetilde{H}_{T}^{q}$ and $\widetilde{E}_{T}^{q}$ defined in [27]. As we saw in Sect. 3.2, their derivation only requires the $x^{n-1}$ moments of the distributions to be polynomials in $\xi$ with maximal power $\xi^{n-1}$. This is indeed the case, as has been shown in 29 .

Gluon helicity-flip distributions appear in DVCS starting at order $\alpha_{s}$, with the hard-scattering formula of the form

$$
\mathcal{F}_{T}^{g}(\xi)=\int_{-1}^{1} d x \frac{1}{\xi} C_{T}^{g}\left(\frac{x}{\xi}\right) \frac{F_{T}^{g}(x, \xi)}{x}
$$

for $F_{T}^{g}=H_{T}^{g}, E_{T}^{g}, \widetilde{H}_{T}^{g}, \widetilde{E}_{T}^{g}$ as defined in [27]. Dispersion representations for this case can be discussed in analogy to the cases considered in the previous sections. To do this requires analysis of the highenergy behavior (see the related work [30] for the helicity-flip structure function $F_{3}^{\gamma}$ of the photon) and of the polynomiality properties (in generalization of the quark case treated in [29]). We shall not do this here.

\section{The model of Freund, McDermott and Strikman}

As an application of the dispersion relations discussed in this work, we now investigate the model for GPDs proposed by Freund, McDermott and Strikman in [5]. We focus on the quark singlet distribution and its generalized counterpart,

$$
\Sigma(x)=\sum_{q}[q(x)+\bar{q}(x)], \quad H(x, \xi)=\sum_{q} H^{q[+]}(x, \xi),
$$

where for ease of notation we have not explicity indicated that $H(x, \xi)$ refers to the quark singlet. Here and in the following we take $t=0$, which does not affect the issue of analyticity to be discussed. In our notation, the model introduced in [5] reads

$$
H(x, \xi)= \begin{cases}\Sigma(x) & \text { for } x \geq \xi \\ \Sigma(\xi) \frac{x}{\xi}\left[1+\frac{15}{2} a(\xi)\left(1-\frac{x^{2}}{\xi^{2}}\right)\right] & \text { for } x<\xi\end{cases}
$$


with $a(\xi)$ chosen to satisfy the polynomiality condition

$$
\int_{0}^{1} d x x H(x, \xi)=\sum_{q} \int_{-1}^{1} d x x H^{q}(x, \xi)=\int_{0}^{1} d x x \Sigma(x)+4 \xi^{2} C_{2}
$$

for the lowest nontrivial Mellin moment, where $C_{2}=\sum_{q} C_{2}^{q}(t=0)$ according to (1). One readily finds

$$
\Sigma(\xi) a(\xi)=\frac{1}{\xi^{2}} \int_{0}^{\xi} d x x \Sigma(x)-\frac{1}{3} \Sigma(\xi)+4 C_{2} .
$$

Clearly, higher Mellin moments of (80) are generally not polynomials in $\xi$ of the order required by (11). At small $\xi$, one may expect that this does not have an important effect on the moments themselves, in the sense that a Taylor expansion

$$
\int_{0}^{1} d x x^{n-1} H(x, \xi)=\sum_{\substack{k=0 \\ \text { even }}}^{\infty}(2 \xi)^{k} A_{n, k}
$$

of a given moment differs from a polynomial of order $\xi^{n}$ by terms vanishing like $\xi^{n+2}$ for $\xi \rightarrow 0$. It is however not obvious that this only leads to small inconsistencies in scattering amplitudes calculated with (80), given that these do not have a simple expression in terms of Mellin moments with integer index $n$.

We have seen that polynomiality of the Mellin moment ensures the consistency of dispersion relations for the hard-scattering kernel and for the process amplitude. Let us check by how much the dispersion representations (27) and (28) differ for the above model. We limit ourselves to the lowest order in $\alpha_{s}$ and take $\operatorname{Im} C^{q[+]}(\omega)=\pi[\delta(\omega-1)-\delta(\omega+1)]$, omitting any global factors in the kernel. The two dispersion representations then read

$$
\begin{aligned}
\operatorname{Re} \mathcal{H}_{d i r}(\xi) & =\int_{0}^{1} d x H(x, \xi)\left[\frac{1}{\xi-x}-\frac{1}{\xi+x}\right] \\
\operatorname{Re} \mathcal{H}_{\xi_{0}}(\xi) & =\int_{0}^{1} d x\left\{H(x, x)\left[\frac{1}{\xi-x}-\frac{1}{\xi+x}\right]+\left[H\left(x, \xi_{0}\right)-H(x, x)\right]\left[\frac{1}{\xi_{0}-x}-\frac{1}{\xi_{0}+x}\right]\right\} .
\end{aligned}
$$

We note that at Born level $\operatorname{Re} \mathcal{H}_{\text {dir }}(\xi)$ calculated from (27) coincides with the real part calculated directly from the factorization formula (17). For a numerical study, we take

$$
x \Sigma(x)=p_{1} x^{-p_{2}}(1-x)^{p_{3}}\left(1+p_{4} x\right)
$$

for the quark singlet distribution, with $p_{1}=0.34, p_{2}=0.25, p_{3}=4, p_{4}=25.4$. This gives a reasonably good approximation of the CTEQ6M distributions at scale $\mu=2 \mathrm{GeV}$. With $p_{3}$ taken as an integer, the integrals required for evaluating (82) and (84) are readily carried out. One finds that $\operatorname{Re} \mathcal{H}_{\text {dir }}\left(\xi_{0}\right)$ diverges for $\xi_{0} \rightarrow \infty$ in this model, so that one cannot use this point for the subtraction required in $\operatorname{Re} \mathcal{H}_{\xi_{0}}$. We take instead the $s$-channel threshold $\xi_{0}=1$, where the model GPD has the simple form $H(x, 1) \propto x\left(1-x^{2}\right)$. As an alternative choice we take the value $\xi_{0}=0.01$ in the small- $\xi$ region. The comparison of the two representations in (84) for several values of $\xi$ is given in Table 3 . We see that their discrepancy is severe and does not improve with decreasing $\xi$. By construction, the two representations coincide of course for $\xi=\xi_{0}$.

The values in the table have been obtained by setting $C_{2}$ to zero in (82). One readily finds that this term gives a contribution of $20 C_{2}$ to both $\operatorname{Re} \mathcal{H}_{\text {dir }}(\xi)$ and $\operatorname{Re} \mathcal{H}_{\xi_{0}}(\xi)$. Taking the value of $C_{2} \approx-0.8$ 
Table 3: The convolution integrals $\operatorname{Re} \mathcal{H}_{d i r}(\xi)$ and $\operatorname{Re} \mathcal{H}_{\xi_{0}}(\xi)$ defined in (84), evaluated for $\xi_{0}=1$ and $\xi_{0}=0.01$. The values are calculated with the GPD model specified by (80) and (82) with $C_{2}=0$. For better legibility, the values of the integrals have been rounded to two significant digits in the first two rows and to the next integer in the remaining ones.

\begin{tabular}{|c|c|c|c|c|c|}
\hline$\xi$ & $\operatorname{Re} \mathcal{H}_{d i r}$ & $\operatorname{Re} \mathcal{H}_{1.0}$ & $\operatorname{Re} \mathcal{H}_{0.01}$ & $\frac{\operatorname{Re} \mathcal{H}_{1.0}}{\operatorname{Re} \mathcal{H}_{d i r}}$ & $\frac{\operatorname{Re} \mathcal{H}_{0.01}}{\operatorname{Re} \mathcal{H}_{d i r}}$ \\
\hline $10^{-4}$ & $12 \times 10^{4}$ & $4.4 \times 10^{4}$ & $4.4 \times 10^{4}$ & 0.37 & 0.37 \\
\hline $10^{-3}$ & $6.5 \times 10^{3}$ & $2.3 \times 10^{3}$ & $2.5 \times 10^{3}$ & 0.35 & 0.39 \\
\hline $10^{-2}$ & 318 & 74 & 318 & 0.23 & 1 \\
\hline 0.1 & 26 & 9 & 253 & 0.37 & 10 \\
\hline 0.3 & 16 & 11 & 255 & 0.70 & 16 \\
\hline 0.5 & 10 & 7 & 251 & 0.76 & 26 \\
\hline
\end{tabular}

estimated in the chiral quark-soliton model [24] would not significantly change the values for small $\xi$, and in any case cannot restore the discrepancy between the two integrals in (84). We must conclude that, even for small $\xi$, the model (80) violates polynomiality and thus Lorentz invariance in a way which leads to serious inconsistencies when using it to calculate the real part of process amplitudes. To obtain consistent results, one may use the ansatz (80) for $|x| \geq \xi$ to calculate $\operatorname{Im} \mathcal{H}(\xi)$ and to restore the real part from the dispersion relation (25), with the subtraction constant left undetermined by the model.

\section{Summary}

Lorentz invariance implies that the Mellin moments of generalized parton distributions are polynomials in the skewness $\xi$ with a maximal power depending on the quantum numbers of the distribution. We have shown that this property leads to integral relations

$$
\int_{-1}^{1} d x F(x, \xi, t)\left[\frac{1}{\omega \xi-x}-\sigma \frac{1}{\omega \xi+x}\right]=\int_{-1}^{1} d x F\left(x, \frac{x}{\omega}, t\right)\left[\frac{1}{\omega \xi-x}-\sigma \frac{1}{\omega \xi+x}\right]+\mathcal{I}(\omega, t)
$$

for $\sigma= \pm 1$ and any $\omega \geq 1$, where $F$ is one of the distributions

$$
H^{q}, E^{q}, \widetilde{H}^{q}, \widetilde{E}^{q}, \quad \frac{H^{g}}{x}, \frac{E^{g}}{x}, \frac{\widetilde{H}^{g}}{x}, \frac{\widetilde{E}^{g}}{x}, \quad H_{T}^{q}, E_{T}^{q}, \widetilde{H}_{T}^{q}, \widetilde{E}_{T}^{q} .
$$

In (86) Cauchy's principal value prescription is to be used at $x= \pm \omega \xi$ and at $x=0$. The only cases where $\mathcal{I}(\omega, t)$ is nonzero occur for unpolarized distributions and $\sigma=+1$, where

$$
\pm \mathcal{I}(\omega, t)=2 \sum_{\substack{n=2 \\ \text { even }}}^{\infty}\left(\frac{2}{\omega}\right)^{n} C_{n}^{q}(t)=2 \int_{-1}^{1} d x \frac{D^{q}(x, t)}{\omega-x} \quad \text { for } F=H^{q}, E^{q},
$$




$$
\pm \mathcal{I}(\omega, t)=4 \sum_{\substack{n=2 \\ \text { even }}}^{\infty}\left(\frac{2}{\omega}\right)^{n} C_{n}^{g}(t)=\frac{2}{\omega} \int_{-1}^{1} d x \frac{D^{g}(x, t)}{\omega-x} \quad \text { for } F=\frac{H^{g}}{x}, \frac{E^{g}}{x}
$$

Here the sign + on the l.h.s. is to be taken for $H^{q}, H^{g}$ and the sign - for $E^{q}, E^{g}$. To establish the relations (86) in the polarized gluon sector, we needed that the moments $\int d x x^{-1} \widetilde{H}^{g}(x, \xi, t)$ and $\int d x x^{-1} \widetilde{E}^{g}(x, \xi, t)$ are independent of $\xi$, and we had to correct the double distribution representation of $\widetilde{H}^{g}$ and $\widetilde{E}^{g}$ used so far in the literature.

For $t \leq 0$ the real part of the leading invariant amplitudes for DVCS or meson production can be obtained from a dispersion relation of the hard-scattering kernel,

$$
\operatorname{Re} \mathcal{F}(\xi, t)=\frac{1}{\pi} \int_{1}^{\infty} d \omega \operatorname{Im} C(\omega) \int_{-1}^{1} d x F(x, \xi, t)\left[\frac{1}{\omega \xi-x}-\sigma \frac{1}{\omega \xi+x}\right]
$$

or for the invariant amplitude itself,

$$
\operatorname{Re} \mathcal{F}(\xi, t)=\frac{1}{\pi} \int_{1}^{\infty} d \omega \operatorname{Im} C(\omega)\left\{\int_{-1}^{1} d x F\left(x, \frac{x}{\omega}, t\right)\left[\frac{1}{\omega \xi-x}-\sigma \frac{1}{\omega \xi+x}\right]+\mathcal{I}(\omega, t)\right\}
$$

where $C=C^{q[\sigma]}, C^{g}$ is the appropriate hard-scattering kernel (for the quark transversity distributions no corresponding process is known). Consistency of the two representations is ensured by (86). The contribution from $\mathcal{I}(\omega, t)$ in (90) is energy independent and can be identified with $\mathcal{F}(\xi, t)$ in the limit $\xi \rightarrow \infty$, i.e. at the point $2 \nu=s-u=0$ below threshold. The corresponding terms given in (88) are due to spin-zero exchange in the $t$-channel. Spin-zero exchange contributions in the parity-odd sector appear in $\widetilde{E}^{q}$ and $\widetilde{E}^{g}$. They do not give a nonzero $\mathcal{I}(\omega, t)$ but can instead generate a term proportional to $\delta(x)$ in $F(x, x / \omega, t)$. In the alternative dispersion representations (58) and (77) for $\operatorname{Re} \xi \mathcal{E}^{q[-]}(\xi, t)$ and $\operatorname{Re} \xi \mathcal{E}^{g}(\xi, t)$ such a $\delta(x)$ term is avoided, and the spin-zero exchange contribution appears directly as a subtraction constant, with

$$
\sum_{\substack{n=1 \\ \text { odd }}}^{\infty}\left(\frac{2}{\omega}\right)^{n} \widetilde{B}_{n, n-1}^{q}(t) \quad \text { or } \quad 2 \sum_{\substack{n=1 \\ \text { odd }}}^{\infty}\left(\frac{2}{\omega}\right)^{n} \widetilde{B}_{n, n-1}^{g}(t)
$$

playing the same role as $\mathcal{I}(\omega, t)$ in (90).

In Sect. 5 we have seen that the relation (86) can be strongly violated in models of GPDs that do not respect polynomiality, even for small $\xi$. In particular, we found that the model proposed in 5 ] leads to serious conflicts with dispersion relations when used for calculating the real part of scattering amplitudes.

The representation (90) has important consequences on the information about GPDs that can be extracted from DVCS and meson production. To leading approximation in $\alpha_{s}$, the imaginary part of the amplitude is only sensitive to the distributions at $x=\xi$, and the only additional information contained in the real part is a constant associated with pure spin-zero exchange, given by (88) or (91) at $\omega=1$. In [4] this was referred to as a holographic property. Beyond leading order the evaluation of both imaginary and real parts of the amplitude involves however the full DGLAP region $|x| \geq \xi$. In addition, the real part depends on the appropriate spin-zero term in (88) or (91) at all $\omega \geq 1$. We remark that in [1] the possibility was discussed to reconstruct the subtraction terms in (88) from the imaginary part of the DVCS amplitude combined with the inclusive deep inelastic cross section. 
Consider the comparison of a given model or parameterization of GPDs with data on DVCS or meson production. In a leading-order analysis (which should of course always be restricted to kinematics where the LO approximation is adequate) it is sufficient to characterize each GPD by its values at $x=\xi$, supplemented by a constant for the spin-zero exchange contribution discussed above. On one hand this can be a welcome simplification, and on the other hand it indicates the limitations of an LO analysis: when confronting data with a given GPD one is sensitive to $x \neq \xi$ (and to the details of the spin-zero exchange contribution) only at NLO or higher accuracy.

Let us finally emphasize that the imaginary part of an amplitude involves GPDs with skewness given by the value of $\xi$ in the measurement, whereas the dispersion representation (90) of the real part involves all values of the skewness from 0 to 1 . For measurements in a limited energy region, the extra information of the real part compared with the imaginary one is thus not limited to the spin-zero exchange terms.

\section{Acknowledgments}

We gratefully acknowledge discussions with I. Anikin, D. Müller and A. Schäfer. D. I. thanks the DESY Theory Group and the Institute for Theoretical Physics at the University of Regensburg for their hospitality. This work is supported by the Helmholtz Association, contract number VH-NG-004, and the work of D. I. is supported in part by grants RFBR-06-02-16064 and NSh 5362.2006.2.

\section{References}

[1] K. Kumerički, D. Müller and K. Passek-Kumerički, hep-ph/0703179.

[2] D. Yu. Ivanov, A. Schäfer, L. Szymanowski and G. Krasnikov, Eur. Phys. J. C 34 (2004) 297 hep-ph/0401131.

[3] J. C. Collins, L. Frankfurt and M. Strikman, Phys. Rev. D 56 (1997) 2982 hep-ph/9611433.

[4] O. V. Teryaev, hep-ph/0510031;

I. V. Anikin and O. V. Teryaev, arXiv:0704.2185 [hep-ph].

[5] A. Freund, M. McDermott and M. Strikman, Phys. Rev. D 67 (2003) 036001 hep-ph/0208160.

[6] X. D. Ji, J. Phys. G 24 (1998) 1181 hep-ph/9807358.

[7] M. Diehl, Phys. Rept. 388 (2003) 41 hep-ph/0307382.

[8] M. V. Polyakov and C. Weiss, Phys. Rev. D60 (1999) 114017 hep-ph/9902451.

[9] I. V. Musatov and A. V. Radyushkin, Phys. Rev. D61 (2000) 074027 hep-ph/9905376.

[10] O. V. Teryaev, Phys. Lett. B510 (2001) 125 hep-ph/0102303.

[11] B. C. Tiburzi, Phys. Rev. D 70 (2004) 057504 hep-ph/0405211.

[12] A. V. Belitsky, D. Müller and A. Kirchner, Nucl. Phys. B629 (2002) 323 [hep-ph/0112108].

[13] J. C. Collins and A. Freund, Phys. Rev. D 59 (1999) 074009 hep-ph/9801262. 
[14] A. Freund and M. F. McDermott, Phys. Rev. D 65 (2002) 074008 hep-ph/0106319.

[15] D. Müller and A. Schäfer, Nucl. Phys. B 739 (2006) 1 hep-ph/0509204].

[16] S. V. Goloskokov and P. Kroll, Eur. Phys. J. C 50 (2007) 829 hep-ph/0611290].

[17] A. V. Belitsky and A. V. Radyushkin, Phys. Rept. 418 (2005) 1 [hep-ph/0504030].

[18] D. Yu. Ivanov, L. Szymanowski and G. Krasnikov, JETP Lett. 80 (2004) 226 hep-ph/0407207;

N. Warkentin, M. Diehl, D. Yu. Ivanov and A. Schäfer, hep-ph/0703148.

[19] E. B. Zijlstra and W. L. van Neerven, Nucl. Phys. B 383 (1992) 525.

[20] S. I. Ando, J. W. Chen and C. W. Kao, Phys. Rev. D 74 (2006) 094013 hep-ph/0602200;

M. Diehl, A. Manashov and A. Schäfer, Eur. Phys. J. A31 (2007) 335 hep-ph/0611101.

[21] M. Guidal and M. Vanderhaeghen, Phys. Rev. Lett. 90 (2003) 012001 hep-ph/0208275];

A. V. Belitsky and D. Müller, Phys. Rev. Lett. 90 (2003) 022001 hep-ph/0210313]; Phys. Rev. D 68 (2003) 116005 hep-ph/0307369.

[22] E. B. Zijlstra and W. L. van Neerven, Nucl. Phys. B 417 (1994) 61, Erratum ibid. B 426 (1994) 245.

[23] L. Mankiewicz, G. Piller and A. V. Radyushkin, Eur. Phys. J. C10 (1999) 307 hep-ph/9812467.

[24] K. Goeke, M. V. Polyakov and M. Vanderhaeghen, Prog. Part. Nucl. Phys. 47 (2001) 401 hep-ph/0106012.

[25] A. V. Belitsky, D. Müller, A. Kirchner and A. Schäfer, Phys. Rev. D 64 (2001) 116002 hep-ph/0011314.

[26] P. Hoodbhoy and X. D. Ji, Phys. Rev. D 58 (1998) 054006 hep-ph/9801369;

A. V. Belitsky and D. Müller, Phys. Lett. B 486 (2000) 369 [hep-ph/0005028].

[27] M. Diehl, Eur. Phys. J. C19 (2001) 485 [hep-ph/0101335].

[28] D. Yu. Ivanov, B. Pire, L. Szymanowski and O. V. Teryaev, Phys. Lett. B 550 (2002) 65 hep-ph/0209300.

[29] Ph. Hägler, Phys. Lett. B 594 (2004) 164 hep-ph/0404138.

[30] B. Ermolaev, R. Kirschner and L. Szymanowski, Eur. Phys. J. C 7 (1999) 65 hep-ph/9806439. 